\title{
LIBANIO Y LA CULTURA LIBRESCA TEMPRANO-BIZANTINA ${ }^{1}$
}

\section{TOMÁs FERNÁNDEZ \\ Conicet-UBA / KU Leuven \\ fernandez_tomas@yahoo.com}

\begin{abstract}
Este artículo pretende dar cuenta del rol de los libros, los copistas y la circulación de textos en la obra de Libanio (s. IV), y especialmente en su Autobiografia (= oratio 1). Sobre la base de las referencias a la cultura libresca, por lo demás, se establecen vínculos con la cultura griega anterior, en particular con la segunda sofística; contemporánea y cristiana; y posterior, de los períodos medio y tardo-bizantino.
\end{abstract}

Libanio / retórica / cultura libresca / Bizancio

\section{LIBANIUS AND EARLY BYZANTINE BOOK CULTURE}

This article tackles the role of books, scribes and text circulation in the œuvre of Libanius (4th c.), especially in his Autobiography (= oratio 1). On the basis of his references to scriptorial culture, I underline his links to previous Greek culture mainly to the Second Sophistic -; to contemporary Christian paideia; and to later Byzantine erudition.

Libanius / rhetoric / scriptorial culture / Byzantium

1 El autor desea agradecer al Conicet, que generosamente financia su investigación sobre la cultura tardo-antigua y bizantina. 
ibanio, "sofista del período temprano-bizantino"2, puede también considerarse un exponente tardío de la segunda sofística, que floreció en los siglos I-III d. $\mathrm{C}^{3}$. Con todo, en los últimos tiempos ha surgido la noción de "tercera sofística", uno de cuyos principales representantes sería, precisamente, Libanio ${ }^{4}$. También esta última conceptualización ha sido combatida: la "tercera sofística" no existiría como movimiento autónomo; tal formulación, por el contrario, no haría más que oscurecer los vínculos que unen a Libanio y demás rétores de los siglos IV y siguientes con los sofistas de los siglos I-III d. $\mathrm{C}^{5}$. Pero los puntos en común entre Libanio y los autores de la segunda sofística no son menos claros que aquellos que lo vinculan con autores posteriores. El gusto extraordinario que el patriarca Focio (s. IX), por ejemplo, sentía al leer las $\mu \varepsilon \lambda \varepsilon ́ \tau \alpha ı$ (declamationes) de Libanio, prefiriéndolas al resto de la obra del sofista ${ }^{6}$, es análogo al que hizo al público lector favorecer las paráfrasis de Simeón Metafrastés (s. X) -refinadas, retóricas y despojadas de detalles materiales e históricos puntuales-, en detrimento de las vidas de santos originales, llanas y sin gran ornato, muchas de las cuales dejaron de ser copiadas y acabaron perdiéndose: es, por ende, un gusto plenamente bizantino.

Un punto privilegiado para explorar la situación histórica de Libanio está constituido por sus referencias a libros, copistas y circulación de textos. Tales referencias, por lo demás, apuntan tanto al pasado como al futuro, ya que en gran medida están dictadas por convenciones de estilo antes que por voluntad de dejar testimonio de un elemento material concreto. En tanto partes del dispositivo retórico, con una función determinada de relativamente escasa autonomía, las alusiones al material escriturario presentan un doble interés, tanto socio-histórico como narrativo-retórico.

\footnotetext{
2 Hunger (1978: 93).

3

4

AMATO et al. (2006)

5 VAN Hoof (2010: passim). Nótese que la autora fuerza las conceptualizaciones de quienes propugnan la existencia de una tercera sofística en la Antigüedad tardía. Así, por ejemplo, resalta que A. Hostein, en una reseña a Amato et al. (2006), "speaks about the autonomy of late ancient rhetoric". Esta afirmación es inexacta. Hostein, a quien la misma Van Hoof cita, se refiere a la autonomía de los estudios sobre retórica antigua, no a la autonomía de la retórica misma: "Employer cette notion revient à accorder aux études sur la rhétorique tardoantique une autonomie, un caractère propre et irréductible [...]", cit. en Van Hoof (2010: 213). Si se pretendiera que la retórica misma, en el siglo V o en el III, está desgajada de su contexto socio-cultural, sin duda habría una falla metodológica grave. Como Hostein se refiere a la autonomía de los trabajos sobre dicha retórica, al contrario de lo que sugiere Van Hoof, no hay nada que objetar.

6 Hunger (1978: 93-94): "So besitzen wir von Libanios noch 51 derartige $\mu \varepsilon \lambda \varepsilon ́ \tau \alpha$, drei volle Bände der Ausgabe von Förster (Bd. 5-7), die Photios mehr schätzte als das übrige OEuvre des Rhetors."
} 
Bajo esta doble perspectiva se analizan a continuación una serie de ejemplos tomados de preferencia de la Autobiografia de Libanio, así como de unas pocas cartas.

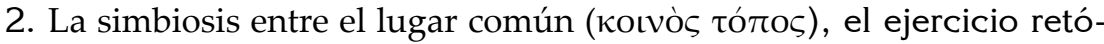
rico (e.g. de la hipérbole) y la "realidad" material se manifiesta con claridad en Autobiografía § 1557. Aquí Libanio, al señalar que necesariamente su éxito genera envidia, observa que las muchas diestras de los copistas ( $\beta \iota \beta \lambda \iota \gamma \varrho \alpha ́(\varphi \circ \iota)$ en realidad resultan pocas, teniendo en cuenta la enormi-

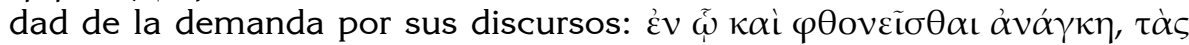

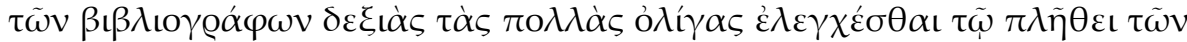
$\dot{\varepsilon} 0 \alpha \sigma \tau \tilde{\omega} v$ [i.e. de sus discursos]. En $\S 113$ señala que nada menos que diez copistas se dedican a transcribir un panegírico suyo, consagrado a un prefecto. Allí dedica una invectiva, conocida entre otros autores antiguos y bizantinos, contra un copista que osa modificar su exemplar, como sucedió con uno de estos diez ${ }^{8}$. Aquí, ambas referencias a $\beta \iota \beta \lambda \iota \gamma \gamma \varrho \alpha ́ \varphi$ o puntuales son ante todo un modo de reforzar un punto retórico: la exaltación de su propio éxito.

Distinto es el caso de una epístola en que pregunta a su interlocutor qué partes de su obra posee, para no fatigar inútilmente a los copistas haciéndolos transcribir algo que el otro individuo ya tiene en su poder ${ }^{9}$. En Ep. 347 señala que estaba a punto de no enviar una carta, entre otros motivos, por la escasez de copistas ( $\gamma \varrho \alpha \varphi \varepsilon i \tilde{\varsigma}$, utilizado cuasi sinonímicamente con

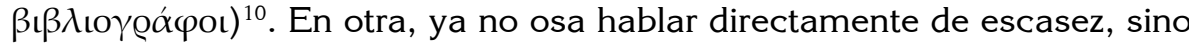
que, mediante un eufemismo que manifiesta su seco humor irónico, deplo-

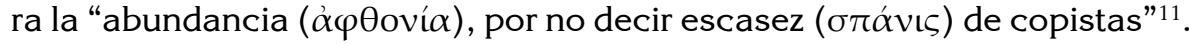
Por lo demás, las cartas no sólo eran enviadas a los destinatarios: Libanio

\footnotetext{
7 Todas las citas de Libanio, incluyendo las de su correspondencia con Basilio de Cesarea, se hacen según la edición de Foerster (1903-1922). Para la Autobiografia, se ha consultado además Norman (1965 y 1992)

8

Estos cambios consistieron en alterar muchas de sus expresiones y agregar pequeñas

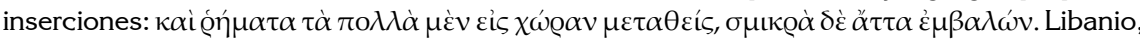
en consonancia con una ideología de persecución a su persona que impregna toda su obra, considera que las alteraciones del copista se deben a las diabólicas maquinaciones de un sofista rival.

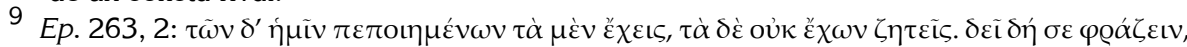

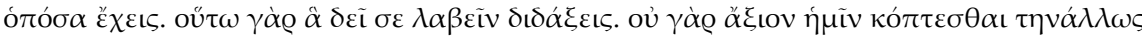

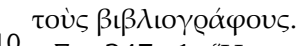

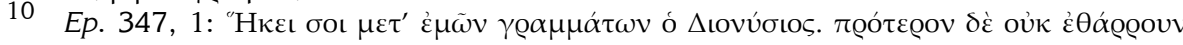

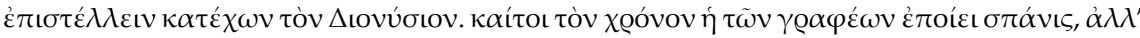

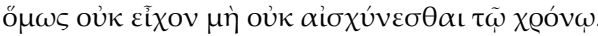

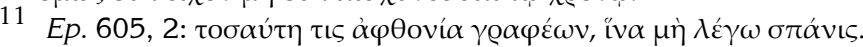


ejecutaba además copias de las mismas (como se deduce de su ofrecimiento de hacer llegar de nuevo una extraviada a uno de sus destinatarios) y, según el insigne estudioso Seeck, probablemente las conservara no en hojas sueltas sino en un libro ${ }^{12}$. Las copias probablemente fueran realizadas por copista, ya que en ocasiones Libanio escribe de su puño y letra. Así, en Autobiografía §103 señala que mientras estaba sentado dedicado a su creación, un tumulto "le detuvo la mano" (con la que escribía) ${ }^{13}$. La anécdota que refiere en $\S 202-203$ es más elaborada: en un momento sufre un ataque mientras escribía en su cama, teniendo a su lado a un médico ${ }^{14}$; a continuación, el médico quiere incitarlo a retomar su actividad para de-

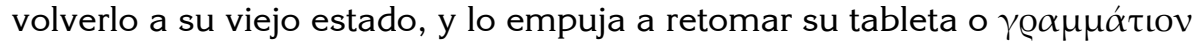
[= $९ \varrho \alpha \mu \mu \alpha \tau \varepsilon i ̃ o v]$, pero es inútil, porque Libanio no recuerda ni su tema ni las palabras que ya llevaba escritas ${ }^{15}$. De todos modos, un buen copista es capaz de producir manuscritos con ventajas técnicas innegables frente a los que podría componer el propio Libanio. En efecto, en Aut. § 232 señala que los $\gamma \varrho \alpha ́ \mu \mu \alpha \tau \alpha$ de un secretario recientemente fallecido contribuían grandemente a sus declamaciones, siendo superiores a los suyos, que obedecían a la necesidad de la producción, mientras los del secretario podían recorrerse con la vista ${ }^{16}$. Podemos imaginar que el manuscrito del secretario estaba dividido en secciones fácilmente visualizables, algo que facilitaba la lectura del conjunto; recordemos las diferencias entre la abigarrada letra cursiva que conservan los códices compuestos por eruditos, y la grafía más elegante de los copistas profesionales.

12 SEECK (1906: 19).

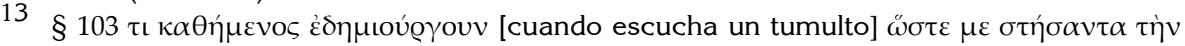
$\chi \varepsilon \tilde{\text { Io } \alpha}[. .$.$] . Otra referencia a su propia mano que escribe en Ep. 17, en que alguien "le$

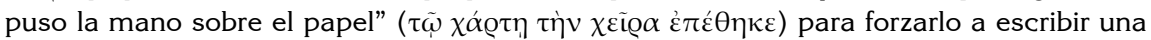
carta aparece. El gesto, que acusa la materialidad de la escritura, también subraya la urgencia que tiene la sugerencia de escribir efectivamente la carta, y se lo menciona, probablemente, para dejar sentada esta compulsión, en parte retórica, en tanto produce un efecto mediante un tópos, a la que Libanio termina cediendo.

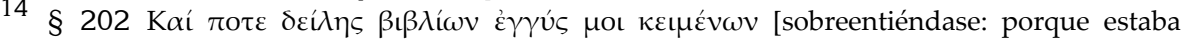

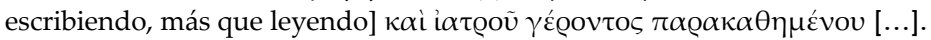

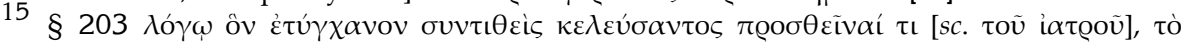

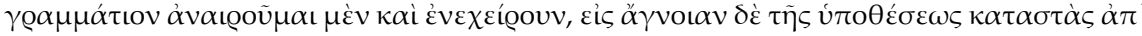

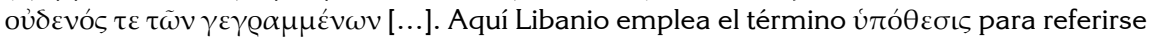
al asunto principal de un discurso, llamado $\theta \dot{\mu} \mu \alpha$ en los manuales retóricos modernos (LAUSBERG 1975: §29); no debe confundirse con la útó $\theta \varepsilon \sigma \iota \varsigma$ en el sentido técnico de respuesta a una quaestio finita (LAUSBERG 1975: § 82, 1).

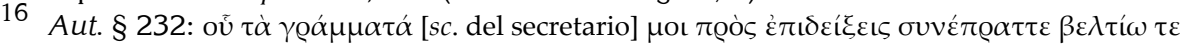

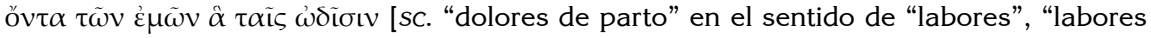

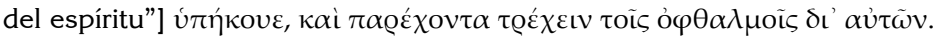


Los copistas atraviesan transversalmente la obra del rétor. En ocasiones se dice de ellos que son dignos de fe, al menos hasta cierto punto. En Ep. 629, Libanio señala que no conoce a quien llevará la carta, pero que su copista Maı́óvı trever, le confía la epístola en cuestión. En una casa como la de Libanio, por lo demás, los secretarios y copistas son también algunos de los principales esclavos y, como tales, están indudablemente sujetos a la tortura ${ }^{17}$. Cuando en una oportunidad las autoridades van a buscar a su secretario

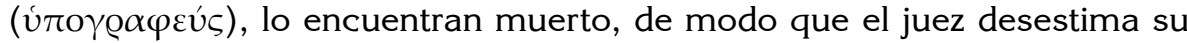
convocatoria: Libanio observa que sólo su muerte lo salvó de la tortura ${ }^{18}$. La interrogación por tortura de los copistas es crónica. En Aut. § 43 un

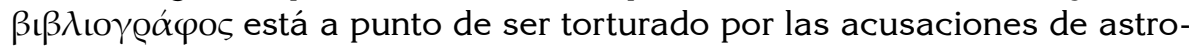
logía y magia hechas contra Libanio por un supuesto envidioso, Bemarco; en Aut. $\S 62$, un sofista planea demandar a Libanio por la enfermedad mental de su esposa (con una acusación de brujería). La tortura, por lo demás, también iba a aplicarse a un adivino (¿un hombre libre?) que había liberado al rétor de sus migrañas; pero nuevamente, y de modo misterioso, el adivino pide a ciertos amigos, entre ellos Libanio, que oren por su

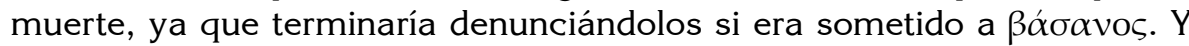
efectivamente el "feliz suceso" se produce a la mañana siguiente: los amigos están celebrando el funeral del adivino cuando llegan las autoridades para prenderlo (Aut. § 173-174). De nuevo, la tortura de los secretarios o copistas (o en este último caso, de un adivino) sirve para resaltar el constante peligro que amenaza al rétor, así como la implacable envidia de sus múltiples enemigos; por vía negativa, le sirve a Libanio para resaltar la grandeza de su propia carrera y su abundancia de méritos, que le acarrea la malevolencia de los desplazados y los incompetentes o, en el caso de los ataques por brujería o magia, de cristianos y paganos.

La magia, que le vale tantos disgustos con la justicia contemporánea, le proporciona también dolores intolerables que de nuevo, en el caso de Libanio, se ligan a la cultura libresca y escrituraria ${ }^{19}$. En Aut. $\S 246$, un embrujo de que ha sido objeto (cf. § 245) hace que renazcan sus crisis de migraña. Le acaece lo que para él es el peor de los males: deja de leer a los antiguos y de escribir y crear discursos, "y tenía disuelto su hablar [en

\footnotetext{
17 Sobre la extensión de la tortura en el Bajo Imperio, cf. HARRIES (1999: 126-127). Los esclavos, evidentemente, seguían siendo pasibles de tortura. Sobre la interrogación por tortura aplicada originalmente a esclavos, cf. GAGARIN (1996: passim).

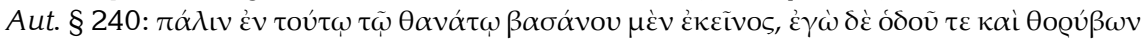

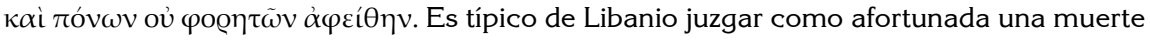
que le ahorra las fatigas del viaje a la sede judicial.

19 Sobre el problema de la magia en Libanio, cf. BonNER (1932); BARB (1993).
} 


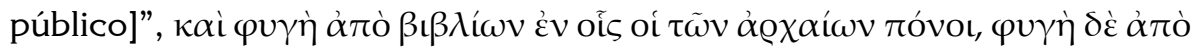

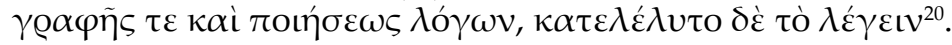

3. Tras este breve recorrido por el papel de copistas y secretarios

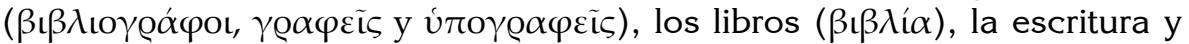

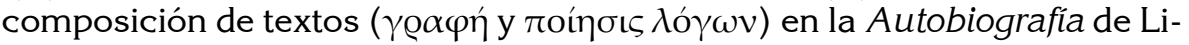
banio, podemos retomar el tema de la continuidad con el pasado (en particular con la segunda sofística) y con los siglos por venir. En esta constelación, no será ocioso recordar los excelentes vínculos epistolares de Libanio con algunos ilustres contemporáneos cristianos, que opera como un reconocimiento de la importancia del nuevo paradigma cultural que no mucho después absorberá por entero a la paideía pagana y que, en esta medida, tiende un lazo sincrónico hacia la cultura del futuro (bizantino) y muestra cuánto esta cultura admiraba la erudición pagana: Basilio de Cesarea concluye una carta con un epigrama laudatorio (en prosa) a Libanio (Epistularum Basilii et Libanii quod fertur commercium 20, 2): ع̌ $\mu \pi v o v v$

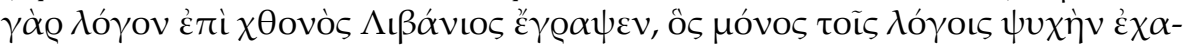
oí $\sigma \alpha \tau$, mientras otro capadocio, Gregorio de Nisa ${ }^{21}$, "parle d'une lettre reçue de Libanius comme d'un 'trésor' mis 'en commun parmi les présents', puisqu'elle avait été lue 'en passant entre les mains de tous'"22. G. Cavallo agrega que muchos copiaron en tablillas la carta para entenderla por entero, algo que también practicaba, por ejemplo, Simplicio, quien creía alcanzar, por el acto mismo de la copia, una comprensión más profunda ( $\alpha \kappa \varrho \iota \beta \varepsilon-$

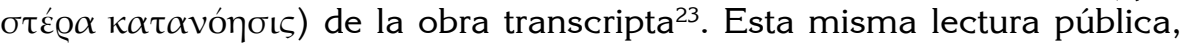
bien atestiguada en autores posteriores, prueba la comunidad de prácticas entre Libanio y los bizantinos medios y tardíos.

En la relación con el pasado, Libanio menciona con devoción a los clásicos, pero no se refiere con frecuencia a los libros materiales ${ }^{24}$. Un caso particular es Aut. $\S 148-150$, en que narra la anécdota del robo y recuperación de un manuscrito de Tucídides que apreciaba enormemente, no sólo por la calidad de la obra, que hubiera podido hacerse copiar de nuevo, sino por la excelencia del códice, de escritura bella y fina y dimensiones reducidas, tanto que podía cargarlo él mismo mientras su

20 Cf. Aut. $\S 268$, en que está a punto de abandonar la enseñanza a causa de migraña.

21

22

23

24 Sí hay una referencia a libros materiales en Aut. § 54, donde señala que le han llegado cantidades de libros ( $\sigma \omega$ ooù $[\ldots] \beta \iota \beta \lambda i \omega v)$. 
esclavo lo seguía ${ }^{25}$ : nunca, agrega Libanio, obtuvo tanto placer de la lectura de Tucídides como cuando lo hacía en esa copia particular ${ }^{26}$. Después de numerosas peripecias, Libanio recupera el códice perdido. La referencia más notable al pasado no es la mención de Tucídides, que podría ser superficial, sino el modo en que modela la narración de la pérdida y recuperación del manuscrito de acuerdo a la estructura de la comedia nueva, como señala Norman: "The text of Thucydides takes on the role of a heroine of New Comedy, experiencing arson (as in Men. Dysc. 60), kidnapping, recognition, restoration"27; el libro mismo reaparece como un hijo largamente perdido que regresa inopinadamente ${ }^{28}$.

Análogo es el caso de los rayos. Sobre Libanio, según su propio relato, caen no en una sino en dos ocasiones. En la primera Libanio, de pie

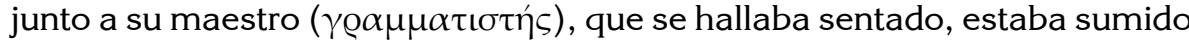
en la lectura de Acarnienses de Aristófanes (guiño a la cultura clásica). En esa circunstancia el cielo se oscurece de golpe y sin que pueda anticipar-

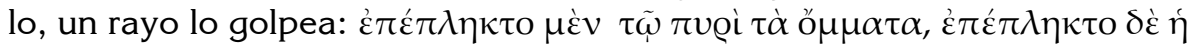

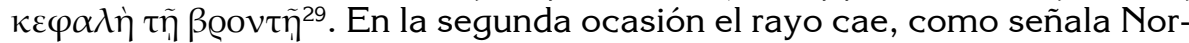
man, "with Homeric force" 30 , por la cita de la Ilíada con que Libanio corona

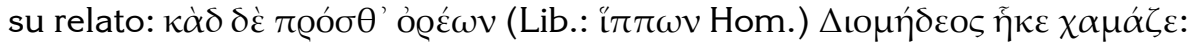
nuevo guiño a los clásicos. Pero, de nuevo, la referencia a la cultura anterior no aparece tanto en las referencias nominales a autores determinados sino en la figuración narrativa de los hechos. Primero, porque Libanio tiene un modelo en el lugar común de los rayos que indican lo extraordinario del carácter sobre el que caen. A título de ejemplo, el nacimiento de Apolonio de Tíana, según lo relata Filóstrato, fue acompañado por un rayo que enviaban los dioses para anticipar su grandeza ${ }^{31}$. Vale la pena recordar que

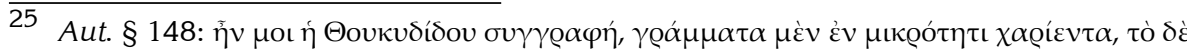

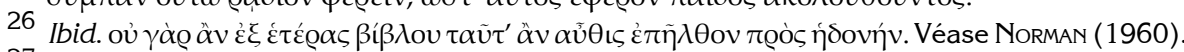

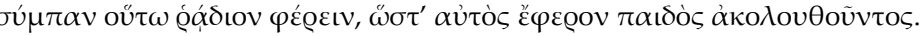

27 Norman (1992: 219, n. a).

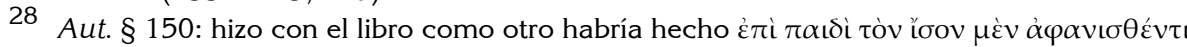

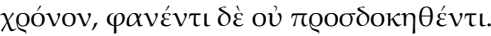

29 Aut. § 9. Cf. Ep. 727, anno 334.

30 Norman (1992: 142, n. b).

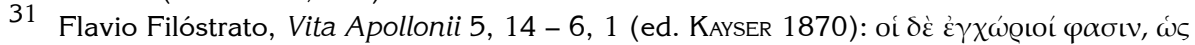

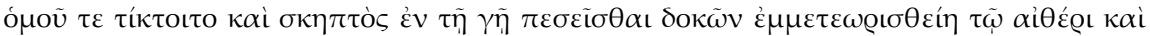

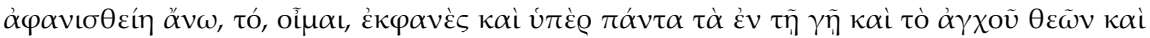

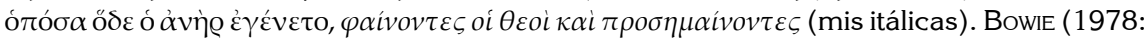
1666-1667) señala que el mismo Filóstrato registra la caída de un rayo también en el nacimiento del sofista Escopeliano, con la salvedad de que en este último caso mueren el gemelo del protagonista y un cierto número de circunstantes. Sobre Apolonio y la biografía de Filóstrato véase la clásica contribución de MEYER (1917). 
el mismo Filóstrato, en su Vida de los sofistas, acuñó la denominación "segunda sofística" en la que no sería inválido situar también a Libanio. Este último autor, por lo demás, no sólo retoma el tópico, invirtiéndolo parcialmente (ya que, tal vez para atenuar los efectos negativos de presentarse como un ser divino, juzga o simula juzgar los incidentes con los rayos como insignes desgracias), sino que lo sobrepuja: sobre él no cae uno sino, como hemos visto, dos rayos. Este sobrepujamiento mismo también es un rasgo de los cultores de los lugares comunes, anteriores o posteriores a Libanio; para una discusión del término, véase el extraordinario capítulo de H.R. Curtius denominado, justamente, "Sobrepujamiento" ${ }^{32}$. La historicidad supuesta o real, en cualquier caso indemostrable, de los rayos que afectaron a Libanio, no le quitan eficacia al lugar común percibido como tal y decodificado en su marco pertinente por un público habituado a las destrezas verbales y las referencias multiformes de Libanio.

4. Un último punto de tensión entre la materialidad cotidiana y el tópos

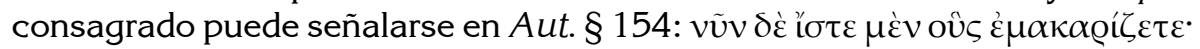

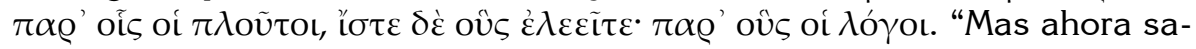
béis a quiénes consideráis dichosos -los que tienen las riquezas- y sabéis

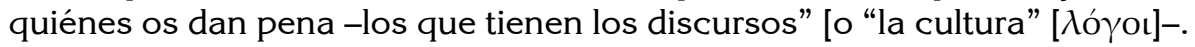
Esto no es más que una reformulación de la tesis inicial de la Autobiografía, figurada como un discurso presentado a un auditorio, y cuyo thema es una demostración, a saber, la de que Libanio no es tan feliz como dicen algunos ni tan infeliz como dicen otros ${ }^{33}$. Tal premisa, vaga y generalísima, permite cualquier desarrollo imaginable, y por eso resulta útil. La crítica a la riqueza de la que carecerían quienes se dedican a los $\lambda o ́ \gamma o \iota$, por lo demás, se inserta perfectamente en ese marco. Aquí hay un contraste con el pasado: los rétores de la segunda sofística tenían posiciones de alta influencia y enormes remuneraciones; los profesores posteriores a $\mathrm{Li}$ banio, en cambio, sufrían la miseria, o al menos eso afirmaban: cuando el professor anonymus de una colección epistolar del s. X conservada en un solo manuscrito, el British Museum Add. 36749, reclama el pago de sus

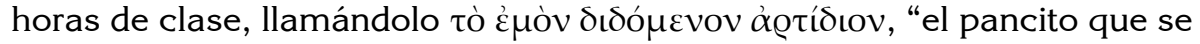
me da" (carta 1), o recibe mal y tarde su salario, si bien, por una parte,

32 CuRtius (1955: 235-239). El tópico de la Überbietung es utilizado por Curtius originalmente en otro sentido (el de las comparaciones explícitas en que se exalta al segundo elemento,

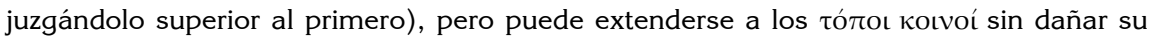
esencia.

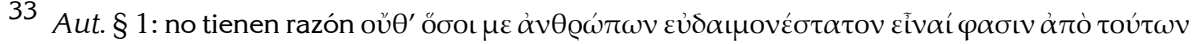

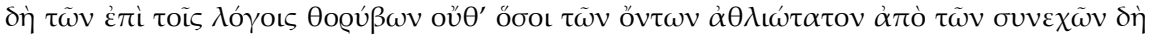

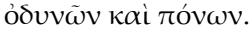


representa su mundo cotidiano, también retoma un tópico que ya aparecía en Libanio ${ }^{34}$. Así deja implícitamente asentada la continuidad de este autor en siglos posteriores, visible en el amor por los alardes y rareza de estilo que también el professor anonymus cultiva. También dan cuenta de esa continuidad los elogios explícitos de un Focio, cuando alaba nominatim al rétor de Antioquía, o un lector anónimo del códice Vaticanus graecus 85, que escribe en los márgenes: “¡Oh, Libanio, qué placer leerte!”35.

Puede ser instructivo observar que la retórica, vinculada a la educación e indispensable para acceder a la administración pública, no puede pretender a ninguna autonomía real. En efecto, sufre la tensión entre un impulso de atemporalidad que le es propio, particularmente a partir de la época de la segunda sofística ${ }^{36}$, y la demanda urgente del entorno, que admira o desprecia las declamaciones y provee de sustento al maestro, sea directamente, sea a través de las autoridades municipales o imperiales. Como se ha intentado demostrar, la Autobiografia de Libanio -de la que se han identificado todas las referencias del mundo de las letras, la cultura libresca y la circulación de textos, complementándolas con algunas menciones del cuerpo epistolar del rétor, incluyendo las respuestas de dos padres capadocios- es un espacio privilegiado para observar dicha tensión, que toma allí una forma levemente diferente: la del lugar común que forma parte de una larga tradición y que no necesariamente deja por ello de ser histórico o, en cualquier caso, no coincide por entero con los lugares comunes abstractos; al presentarse formalmente como vividos personalmente, generan un efecto afectivo particular en un público dado, algo que, por lo demás, no implica afirmar que sean percibidos o aceptados como reales. Los ejemplos tomados del mundo de la cultura libresca han sido adecuados, esperamos, para brindar una demostración en este sentido. Así, pretende colmar una pequeña parte de una laguna en el campo de la retórica en general y en el de los estudios sobre Libanio en particular.

34 El cuerpo epistolar ha sido editado en Markopoulos (2000). Una discusión del contexto de las epístolas puede verse en Lemerle (1971: 246-253, con discusión sobre los problemas

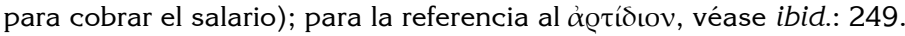

35 Ff. 114r y 224v, cit. en Cavallo (2006: 136).

36 Cf. la debilidad de ciertos rétores por la quaestio infinita, de la que se burla Petronio en los primeros capítulos conservados del Satyricon, del estilo: “¿Qué sucedería si unos piratas secuestran a nuestra hija en su noche de bodas?” o “¿cuál sería la conducta adecuada si para salvar a la patria debiéramos degollar a nuestra propia madre?”. Para la ahistoricidad de los escritores imperiales, véase Hopkinson (1994: 3-6), quien, de todos modos, incurre en generalizaciones no siempre adecuadas. 


\section{Bibliografía}

\section{Fuentes primarias}

Foerster, R. (ed.) (1903-1922) Libanii opera, 11 vols.; Autobiographia (= Or. 1), vol. 1, 1903; Epistulae, vols. 10-11, 1921-1922; Epistularum Basilii et Libanii quod fertur commercium, vol. 11, 1922.

Hopkinson, N. (ed.) (1994) Greek Poetry of the Imperial Period, Cambridge. Kayser, C.L. (ed.) (1870) Vita Apolonii, en Flavii Philostrati opera, vol. 1, pp. 1-344, Berlin.

Markopoulos, A. (ed.) (2000) Anonymi Professoris Epistulae, Berlin.

Norman, A.F. (ed.) Libanius' Autobiography (Oration 1), London.

Norman, A.F. (ed.) (1992) Libanius. Autobiography and Selected Letters, vol. 1, Cambridge.

PAsqual, G. (ed.) (1959) Epistulae. Gregorii Nysseni opera, vol. 8.2, Leiden. (Segunda ed.)

\section{Bibliografía secundaria}

Amato, E., Roduit, A. y SteinRück, M. (eds.) (2006) Approches de la Troisième Sophistique. Hommages à Jacques Schamp, Bruxelles.

Barb, A.A. (1963) "The Survival of the Magic Arts", en Momigliano, A. (ed.) The Conflict between Paganism and Christianity in the Fourth Century, Oxford, pp. 100-125.

Bonner, C. (1932) "Witchcraft in the Lecture Room of Libanius", Transactions and Proceedings of the American Philological Association, 63, pp. 34-44.

BowIE, E.L. (1978) "Apollonius of Tyana: Tradition and Reality", en Aufstieg und Niederstand der römischen Welt, II.16.2, pp. 1652-1699.

Cavallo, G. (2006) Lire à Byzance, Paris.

Curtius, H.R. (1955) Literatura europea y Edad media latina, vol. 1, México. (Primera ed. alemana 1948.)

Harries, J. (1999) Law and Empire in Late Antiquity, Cambridge.

Hunger, H. (1978) Die hochsprachliche profane Literatur der Byzantiner, München.

Gagarin, M. (1996) "The Torture of Slaves in Athenian Law", Classical Philology, 91. 1, pp. 1-18.

Lausberg, H. (1975) Elementos de retórica literaria, Madrid. (Primera ed. alemana 1963.) 
LemerLe, P. (1971) Le premier humanisme byzantin. Notes et remarques sur enseignement et culture à Byzance des origines au $X^{e}$ siècle, Paris. MEYER, E. (1917) "Apollonius von Tyana und die Biographie des Philostratus", Hermes 52, pp. 371-424.

Norman, A.F. (1960) "The Book Trade in Fourth-Century Antioch", The Journal of Hellenic Studies, 80, pp. 122-126.

SEECK, O. (1906) Die Briefe des Libanius zeitlich geordnet, Leipzig.

VAN Hoof, L. (2010) "Greek Rhetoric and the Later Roman Empire. The bubble of the 'Third Sophistic'”, Antiquité tardive, 18, pp. 211-224.

Fecha de recepción: 30-03-2014

Fecha de aceptación: 02-04-2014 